\title{
Review of: "The natural compound TMYX reduces SAN cells rate by antagonizing the CAMP modulation of $f$-channels"
}

Elisabetta Cerbai ${ }^{1}$

1 University of Florence

Potential competing interests: The author(s) declared that no potential competing interests exist.

An interesting and well performed study. All experiments were carried out in naive rabbit SAn cells; it would be interesting to assess the effect on different heterologously expressed HCN isoforms for experimental reasons (i.e., without interference by intracellular cAMP) and herapeutic applications (e.g., DRG cells expressing HCN1 isoforms). 